\title{
Intervenção precoce nas dificuldades de aprendizagem da leitura com recurso ao software Graphogame ${ }^{1}$
}

\section{Early intervention in reading difficulties using the Graphogame software}

Ana Sucena ${ }^{2}$, Ana Filipa Silva ${ }^{3}$, Fernanda Leopoldina Viana ${ }^{4}$

\begin{abstract}
Este trabalho foi financiado por Fundos Nacionais através do Instituto Politécnico do Porto/Banco
Santander-Totta e da FCT (Fundação para a CiênSantander-Totta e da FCT (Fundação para a Ciên-
cia e a Tecnologia) e co-financiado pelo Fundo cia e a Tecnologia) e co-financiado pelo Fundo
Europeu de Desenvolvimento Regional (FEDER) através do COMPETE 2020 - Programa Operaciono âmbito do CIEC (Centro de Investigąấo en Estudos da Criança da Universidade do Minho) com a referência POCl-01-0145-FEDER-007562. Expressamos o nosso agradecimento aos professores, pais e crianças das escolas que participaram neste estudo, bem como ao Departamento de Doutora em Psicologia pela PPorto. E P Portuga Adjunta na Escola Superior de Saúde do Politécnico do Porto. É autora de dois testes de avaliação psicológica: TIL - Teste de Idade de Leitura e Alepe - Bateria de Avaliação da Leitura em Portugués (publicados em Sucena e Castro, 2010 e

E-mail: sucena.ana@gmail.com

Fala pela Escola Superio de Saúde do Politécnico do Porto. É terapeuta da tala na Camara Municipal do Porto, Departamento de Educação.

E-mail: afilipa.cs@gmail.com

Doutora em Psicologia pela Universidade do Minho (Portugal). E prolessora associada no Instituto de vários testes de avaliação psicológica (publicados entre 2010 e 2014) e um capítulo da obra $O$ ensino da leitura: a avaliaçấo (Viana, 2009), editado pelo Ministério da Educaçãa.

E-mail: fviana@ie.uminho.pt
\end{abstract}

RESUMO: Neste estudo avaliou-se o impacto de um software de apoio às dificuldades de aprendizagem da leitura - o Graphogame - ao nível das relações letra-som, consciência fonémica, leitura de palavras e leitura de pseudopalavras. Os participantes eram 57 crianças, a frequentar o $1^{\circ}$ ano de escolaridade, falantes nativos do português europeu, identificadas como em risco de virem a apresentar dificuldades de aprendizagem da leitura. As crianças foram divididas em três grupos: (a) intervenção com recurso a software - treino com software Graphogame, (b) intervenção mista treino com recurso ao software Graphogame e a sessões de competências pré-leitoras e leitoras orientadas por técnico de educação e (c) ausência de intervenção para lá daquela prevista no sistema regular de ensino. Os resultados revelam efeitos positivos de ambos os tipos de intervenção em relação ao grupo controlo. Revelam ainda um efeito mais pronunciado para o grupo de intervenção mista do que para o grupo de intervenção com recurso exclusivo ao software.

PALAVRAS-CHAVE: Aprendizagem da leitura; Software de apoio à leitura; Dificuldades de aprendizagem; Graphogame.

ABSTRACT: This study evaluated the impact of a reading intervention software - the Graphogame - on letter-sound relationship, phonemic awareness, word reading and nonword reading. Participants were 57 1st graders, native speakers of European Portuguese, identified as at risk for developing difficulties in reading acquisition. Children were divided into three groups: (a) intervention using the software - training with Graphogame software, (b) combined intervention - training using the Graphogame software and sessions for pre-reading and reading competence supervised by an education technician (c) no intervention beyond that provided for in the mainstream school system. The results show beneficia effects of both types of intervention compared to the control group. Results also reveal a more positive effect for the combined intervention group than for the group that used exclusively the software.

KEYwoRDS: Reading acquisition; Reading support software; Learning difficulties; Graphogame. 


\section{Introdução}

ara aprender a ler a criança necessita de desenvolver duas competências alicerce: a consciência fonémica e as relações letra-som (SEYMOUR e EVANS, 1999; SEYMOUR, ARO e ERSKINE, 2003; SUCENA, CASTRO e SEYMOUR, 2009; VIANA e SUCENA, 2014). A capacidade para analisar os sons que constituem as palavras contribui para que consiga estabelecer mais facilmente as correspondências entre fonemas e as letras correspondentes. É com base nas competências alicerce que começará a desenvolver-se a competência de descodificação que permitirá a leitura de palavras e pseudopalavras de ortografia simples. A investigação revela que até ser atingido um conhecimento de cerca de $80 \%$ das relações entre letras e sons, os resultados de leitura isolada de palavras ou pseudopalavras não atingem os 50\% de respostas corretas. Após atingido esse limiar, a leitura de palavras e de pseudopalavras isoladas ultrapassa os 50\% de sucessos (SUCENA, 2005; SERRANO et al. 2011). O grau de complexidade quanto às relações entre sons da fala e grafemas varia muito consoante a ortografia. Há ortografias consideradas mais transparentes, enquanto outras são consideradas mais opacas. As mais opacas apresentam uma maior complexidade nas relações entre os sons da fala e os grafemas, como é o caso do inglês. As ortografias transparentes têm uma representação próxima de um-para-um entre grafemase fonemas, como é o caso do castelhano. No caso da ortografia portuguesa, o processo de consolidação das relações entre grafemas e fonemas não é muito simples, uma vez que existem diversos casos em que o mesmo grafema representa mais do que um som e outros em que o mesmo som é representado por mais do que um grafema. O português europeu é considerado uma ortografia de grau intermédio (SEYMOUR, ARO e ERSKINE, 2003; VELOSO, 2005).

Independentemente de aprenderem a ler numa ortografia mais ou menos transparente, algumas crianças revelam dificuldades na aprendizagem das relações entre letras e sons. É atualmente consensual que este grupo de crianças apresenta um risco acrescido de vir a revelar dificuldades na aprendizagem da leitura (LYYTINEN, 2008). Atualmente, prevalece a perspetiva segundo a qual se considera prioritário prevenir (em vez de remediar) as dificuldades de aprendizagem da leitura. Idealmente a identificação de crianças em risco de fracassar na aprendizagem da leitura e da escrita deve ocorrer no último ano do ciclo pré-escolar ou logo no início do $1^{\text {o }}$ ano, de forma a que possam ser implementados programas intencionalizados de promoção de competências leitoras básicas. (HATCHER, HULME e SNOWLING, 2004; WIMMER e MAYRINGER, 2002; SAINE, LERKKANEN, AHONEN, TOLVANEN e LYYTINEN, 2011) A identificação e intervenção atempadas evitam as consequências normalmente associadas ao insucesso, como a baixa motivação, as retenções ou o abandono escolar (LYYTINEN, 2008).

As crianças em risco de virem a desenvolver dificuldades de aprendizagem revelam dificuldades em acompanhar o ambiente habitual das escolas, em que o ensino decorre no contexto de turma alargada (em Portugal com um número mínimo de 20 e máximo de 26). Todavia respondem bem à intervenção individualizada, necessitando de múltiplas repetições no treino da competência de descodificação (TORGESEN, 2005).

Os programas de apoio à aprendizagem da leitura com resultados mais promissores combinam o treino explícito ao nível da consciência fonológica com instrução da leitura altamente estruturada (e.g., HATCHER, HULME e ELLIS, 1994; HATCHER et al., 2006). Lovett e colaboradores (2003) enfatizam a necessidade de intervenção direta ao nível dos défices de consciência fonológica, instrução sistemática e explícita ao nível das correspondências entre letras e conjuntos de letras e sons correspondentes e reforço do reconhecimento de palavras, através da leitura de textos construídos com vocabulário controlado. Importa ainda salientar que é possível prevenir 
o insucesso das crianças em risco de virem a apresentar dificuldades de aprendizagem da leitura através da instrução explícita das correspondências letra-som (FOORMAN, FRANCIS, FLETCHER, SCHATSCHNEIDER e MEHTA, 1998). Apesar de o conhecimento relativo às competências de base subjacentes à aprendizagem da leitura estar bem consolidado, o sistema de ensino regular tende a falhar no apoio às crianças em risco de virem a apresentar dificuldades de aprendizagem, no sentido em que não lhes são dadas oportunidades suficientes para a aprendizagem das relações letrasom de modo a poderem desenvolver a fluência de leitura e uma escrita correta (e.g., HATCHER, HULME e SNOWLING, 2004).

Um estudo com crianças identificadas como em risco de virem a apresentar dificuldades na aprendizagem da leitura comparou dois tipos de intervenção: intervenção exclusivamente orientada por um técnico de educação e intervenção combinada, i.e., orientada por um técnico de educação e com recurso a um software de apoio à aprendizagem da leitura, o Graphogame (SAINE, LERKKANEN, AHONEN, TOLVANEN e LYYTINEN, 2011). Este estudo procurava confirmar os dados da investigação relativos ao sucesso das intervenções dirigidas às dificuldades de aprendizagem da leitura adotando sofwares de apoio. De facto, até à primeira década de 2000, apesar de a investigação relatar resultados positivos de intervenções apoiadas por software educativo (e.g., BLOK, OOSTDAM, OTTER e OVERMAAT, 2002; ELBRO, RASMUSSEN e SPELLING, 1996; JIMÉNEZ et al., 2007; MAGNAN e ECALLE, 2006; NICOLSON, FAWCETT e NICOLSON, 2000; REGTVOORT e VAN DER LEIJ, 2007; SOE, KOKI e CHANG, 2000; VAN DAAL e REITSMA, 2000; WISE, RING e OLSON, 1999), a interpretação e a generalização dos resultados foi questionada em virtude de fragilidades metodológicas. Entre outros aspetos, os estudos realizados entre os anos 90 do sáculo XX e a primeira década do século XXI apresentavam falhas, tais como a ausência de grupo de controlo e/ou de estudos de follow up. O estudo de Saine e colaboradores (2011), com desenho longitudinal, propôs-se ultrapassar essas falhas, incluindo a comparação com um grupo de controlo e um estudo de follow up. Os autores concluíram que as crianças que foram alvo de uma intervenção baseada apenas em sessões de apoio individual melhoraram as suas competências. No entanto, o grupo de crianças que usufruiu da intervenção combinada (software e apoio individual orientado por técnico de educação) melhorou de forma mais significativa, não apenas ao nível do conhecimento das relações letra-som e da descodificação, mas também ao nível da fluência e da escrita de letras.

0 Graphogame (GG) é um software de apoio à aprendizagem da leitura e da escrita, que tem como objetivos o treino das relações grafema-fonema com crianças em risco de virem a experienciar dificuldades de aprendizagem da leitura em ambiente amigável de jogo de computador. Atendendo à idade em que se aprende a ler, as intervenções devem ter um caráter lúdico, motivador, desafiante e reforçador, caraterísticas que estão presentes no Graphogame. 0 software foi desenvolvido por investigadores da Universidade de Jyväskylä (Finlândia) com o objetivo de ser uma ferramenta complementar ao ensino regular (LYYTINEN, ERSKINE, KUJALA, OJANEN e RICHARDSON, 2009; LYYTINEN, RONIMUS, ALANKO, POIKKEUS e TAANILA, 2007). O Graphogame funciona com o uso de auscultadores, ouvindo a criança um som correspondente a uma letra (ou palavra/pseudopalavra, em níveis mais avançados) e surgem diversas opções no ecrã. A criança terá de selecionar a opção escrita correspondente ao som. A promoção da consciência fonémica é assegurada pela exposição simultânea a letras (visualmente) e fonemas (auditivamente). O jogo contribui para a aprendizagem da leitura por apresentar os mesmos estímulos centenas de vezes, em diferentes níveis e em diferentes tarefas. Além disso, o jogo está desenvolvido para que a criança faça associações rápidas, de forma a promover a automatização da leitura. Ao longo do jogo surgem dois jogos tipo: as tarefas principais que exigem associação de um 
segmento áudio à representação visual (cf. Fig. 1) e as tarefas mais ativas que exigem a escrita de uma palavra ou pseudopalavra ouvida (cf. Fig. 2 e 3).

Figura 1 - Exemplo de um cenário de uma tarefa principal do GG

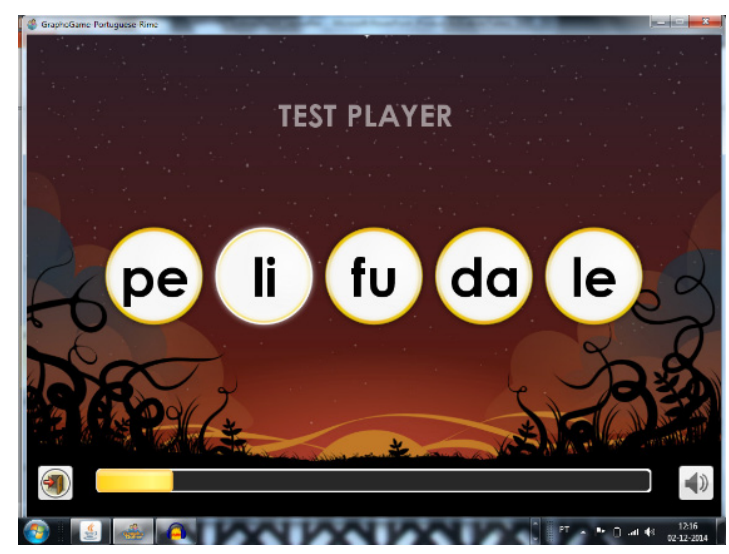

Fonte: Graphogame

Figura 2 - Exemplo de um cenário de uma tarefa mais ativa do GG.

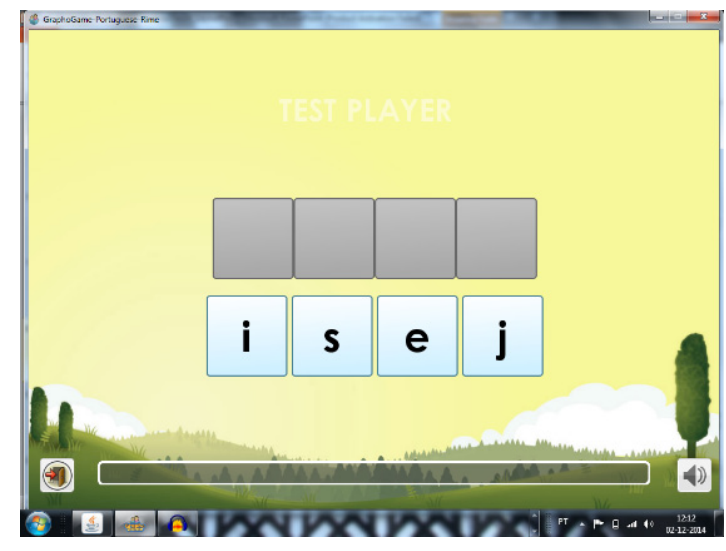

Fonte: Graphogame
Figura 3 - Leitura de palavras em dois cenários - jogo da escada e do submarino

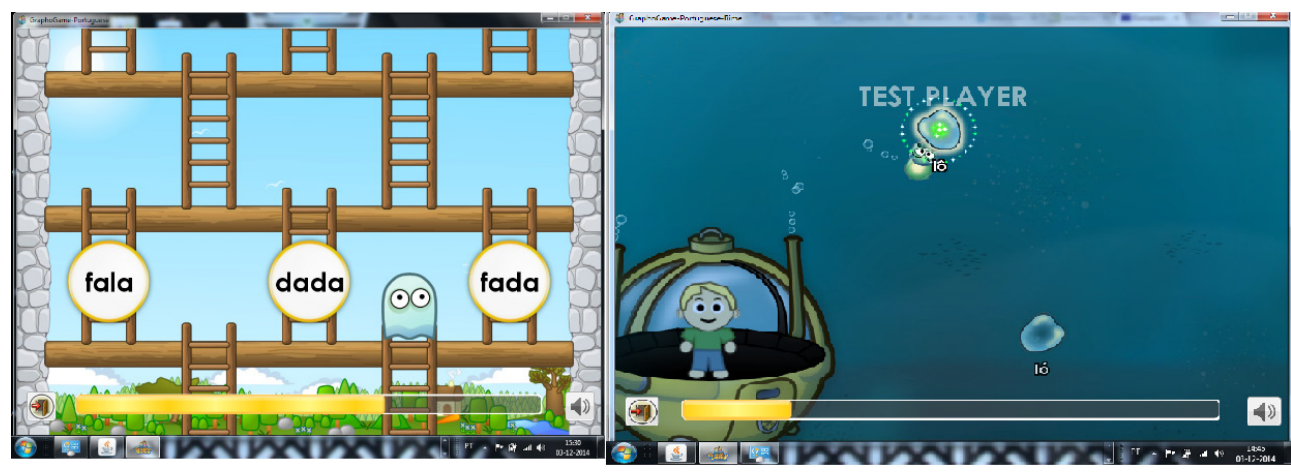

Fonte: Graphogame

Este software de apoio à aprendizagem da leitura tem sido adaptado para diversas línguas (inglês, alemão, português entre outras) com resultados que indicam tratar-se de uma ferramenta eficaz no apoio à aprendizagem da leitura e da escrita. Um estudo conduzido com crianças finlandesas revelou um progresso significativo ao nível do conhecimento de letras, da leitura e de competências de escrita. No estudo de follow up (após 16 meses) as crianças do grupo experimental apresentavam resultados semelhantes às crianças sem dificuldades quer ao nível da precisão e fluência da leitura, que ao nível da escrita (KYLE, KUJALA, RICHARDSON, LYYTINEN e GOSWAMI, 2013). Na Áustria (Graphogame Alemão), crianças a frequentar o $2^{\circ}$ e $4^{\circ}$ anos melhoraram a precisão e a velocidade da leitura após seis semanas de intervenção (HUEMER, LANDERL, ARO e LYYTINEN, 2008). No Reino Unido, após doze semanas de intervenção, as crianças do grupo experimental melhoraram na leitura, na escrita e nas competências fonológicas em comparação com as crianças do grupo controlo. Passados quatro meses da intervenção os ganhos ainda se mantinham (KYLE et al., 2013). Em Portugal foi realizado um estudo de avaliação do impacto do Graphogame Português Alicerce com 
38 crianças, ao longo de 16 semanas (SUCENA, SILVA, CRUZ e VIANA, no prelo). Os resultados revelaram um efeito de aprendizagem mais pronunciado no grupo experimental do que no grupo controlo. Ao nível das competências pré-leitoras os resultados foram mais elevados para o grupo experimental do que para o grupo controlo. Nas competências da leitura (mais especificamente, as competências de descodificação, avaliadas pela leitura de palavras e pseudopalavras) as crianças do grupo experimental obtiveram melhores resultados do que as crianças do grupo controlo. No entanto, apesar da progressão ser significativa, os resultados não foram além dos $50 \%$

O objetivo do presente estudo é avaliar o impacto de dois tipos de intervenção: uma intervenção exclusivamente baseada no recurso diário ao software Graphogame, vantajosa do ponto de vista do tempo despendido e da pouca exigência ao nível de recursos humano, e já com resultados comprovados (SUCENA et al., no prelo), e uma intervenção mista, com recurso diário ao software Graphogame e apoio semanal (uma sessão de trinta a quarenta minutos) com atividades lúdicas para a promoção das competências pré-leitoras e leitoras. Efetivamente, ao longo do estudo piloto relativo à avaliação do Graphogame, foi detetado o elevado potencial de identificação de dificuldades com base na observação da criança a jogar o Graphogame, dificuldades nem sempre ultrapassáveis com o progredir do jogo. De modo a garantir a vertente lúdica, o Graphogame foi programado para persistir um número limitado de vezes nos mesmos estímulos e jogos, após o que a criança "passa" à etapa seguinte. Esta metodologia resulta com grande parte das crianças, mas não é tão eficaz com as crianças com competências mais frágeis que, por vezes, avançam para níveis de treino para os quais não têm as bases suficientes, beneficiando cada vez menos do jogo. Com base nesta observação e nos resultados da investigação (e.g., SAINE, 2011) pretende-se verificar se o efeito da intervenção mista é superior ao efeito da intervenção realizada exclusivamente com recurso ao Graphogame.

\section{Materiais e Métodos}

Foram avaliadas cinquenta e sete crianças a frequentar o primeiro ano de escolaridade em escolas públicas do distrito do Porto, norte de Portugal. As crianças foram selecionadas por indicação do professor e com base nos resultados obtidos num conjunto de provas que apontavam para a existência de risco de experimentarem dificuldades na aprendizagem da leitura, mas sem qualquer necessidade educativa adicional. Para poderem participar no estudo, foi requerida autorização escrita por parte das autoridades escolares e dos pais. Neste estudo procedeu-se à comparação de dois tipos de intervenção em contraste com o grupo controlo. Além das atividades normais previstas no sistema de ensino, o grupo de intervenção 1 (intervenção mista), composto por vinte e quatro crianças, foi submetido ao treino diário com o Graphogame e, uma vez por semana, a exercícios de promoção de competências pré-leitoras e leitoras. O grupo de intervenção 2 (intervenção Graphogame), constituído por vinte crianças, foi submetido exclusivamente à intervenção com o Graphogame. Finalmente o grupo 3 (controlo), com treze crianças, não sofreu qualquer intervenção para lá das atividades escolares previstas no sistema de ensino português (cf. Tab. 1).

Tabela 1 - Descrição dos participantes por grupo

\begin{tabular}{lccc}
\hline \multicolumn{1}{c}{ Características } & Grupo 1 & Grupo 2 & Grupo 3 \\
$\mathrm{N}$ & 24 & 20 & 13 \\
Género (F ; M) & $12 ; 12$ & $9 ; 11$ & $4 ; 9$ \\
Idade (anos ; meses) & $6 ; 4$ & $6 ; 7$ & $7 ; 0$ \\
\hline
\end{tabular}


As crianças dos grupos 1 e 2 (respetivamente, intervenção mista e intervenção Graphogame) foram avaliadas antes da intervenção (M1, préteste, respetivamente, fevereiro de 2016 e outubro de 2015 e) e novamente ao final de dezasseis semanas de intervenção (M2, pós-teste, respetivamente março de 2016 e junho de 2015). As crianças do grupo 3 (controlo) foram avaliadas exatamente nos mesmos momentos que o grupo 2. Nos dois momentos, as avaliações foram realizadas individualmente em contexto escolar.

Na primeira avaliação, todas as crianças foram avaliadas através do teste Matrizes Progressivas Coloridas de Raven (adaptação portuguesa de Simões, 2003), medida de inteligência não-verbal, de modo a excluir problemas de índole cognitiva. Nenhuma criança obteve resultados abaixo do que é considerado capacidade intelectual média. Este teste consiste na apresentação de figuras com um padrão lógico, onde uma das partes é deixada em branco e a criança tem que escolher a figura correta de um conjunto de seis opções a que completa o padrão. Os resultados brutos são depois transformados em percentis.

As crianças foram avaliadas quanto à identificação de letras, escrita de letras, consciência fonémica, leitura de palavras e de pseudopalavras, através de subtestes da bateria de avaliação da leitura ALEPE - Avaliação da Leitura em Português Europeu (SUCENA e CASTRO, 2011).

Na tarefa de identificação de letras, a tarefa da criança consistia em ler uma lista de letras (minúsculas) apresentadas no ecrã do computador. São aceites como respostas corretas quer o ou um dos valores que a letra pode assumir, quer o nome da letra. Por exemplo, para a letra F são cotadas como corretas quer a resposta /fe/ quer a resposta /éfe/.

Na tarefa de escrita de letras a criança tinha de escrever as letras ditadas pelo examinador (em formato minúsculas). Este subteste é constituído por dois itens de treino e vinte e três itens que correspondem à tarefa. 0 resultado total responde ao número total de letras escritas corretamente pela criança. $\mathrm{O}$ tempo despendido na tarefa não é contabilizado. $\mathrm{O}$ teste foi aplicado seguindo as instruções do respetivo manual.

Quanto à tarefa de consciência fonémica, a criança tinha de ser capaz de identificar o fonema comum num par de palavras. Este subteste é constituído por três itens de treino e doze itens que correspondem à tarefa. Cada item é formado por um par de palavras, que se encontra identificado segundo a sua estrutura silábica, nomeadamente CV [Consoante-Vogal (sílaba aberta) + CVC [Consoante-Vogal-Consoante (sílaba fechada)]. O resultado corresponde ao total de respostas corretas.

Nas tarefas de leitura de palavras e de pseudopalavras a criança era convidada a ler uma lista de palavras apresentadas de forma isolada no ecrã do computador. Das duas listas de palavras que fazem parte deste teste, aplicou-se a Lista $\mathrm{A}$, destinada às crianças a frequentar o primeiro ano de escolaridade ou de nível de leitura equivalente. A Lista A é constituída por quatro itens de treino e dezoito itens que correspondem à tarefa. Os itens são constituídos por palavras simples, consistentes e inconsistentes do ponto de vista ortográfico, e por pseudopalavras simples e consistentes. Os resultados obtidos são apresentados em percentagem de acertos por condição ortográfica e também por tempo de reação. O software ALEPE contabiliza o Tempo de Reação entre a apresentação de cada um dos estímulos e a sua leitura. Caso o tempo de reação ultrapasse os 10 segundos, o software considera a resposta como ausente.

A intervenção com o grupo 1 incluía o recurso ao software Graphogame com uma periodicidade de quatro sessões por semana, com uma duração entre dez quinze minutos, e uma sessão semanal de 30 a 40 minutos dinamizada por um profissional especialmente preparado (terapeuta da fala, psicólogo ou professor). Nessa sessão eram realizadas atividades de estimulação de dois tipos de competências: (a) pré-leitoras, como jogos de associação entre 
letras e sons ou de consciência fonémica e; (b) atividades de descodificação e de escrita, com recurso a materiais de caráter lúdico, criados especificamente para o presente estudo. Na intervenção junto do grupo 2, as crianças jogaram o Graphogame entre 10 a 15 minutos por dia, cinco dias por semana, igualmente sob a supervisão de um profissional especialmente preparado. A duração da intervenção com o Graphogame - entre 10 e 15 minutos - está de acordo com a prática usada noutras investigações realizadas com recurso a este software (KYLE, KUJALA, RICHARDSON, LYYTINEN e GOSWAMI, 2013). Esta duração permite que a criança mantenha níveis elevados de motivação e de atenção durante o tempo de jogo e mantenha a motivação na transição de dia para dia. Finalmente, as crianças do grupo 3 (controlo) seguiram o percurso escolar normal.

\section{Resultados}

Os resultados dos três grupos de crianças são apresentados na Tabela 2 com valores em percentagem de respostas corretas. Em todas as medidas verificou-se um efeito de aprendizagem mais pronunciado para o grupo 1 (intervenção mista) face aos grupos 2 (intervenção Graphogame) e 3 (controlo). Verificou-se ainda um efeito de aprendizagem mais acentuado para o grupo 2 (intervenção Graphogame) em relação ao grupo 3 (controlo).

Dado que não se encontravam reunidos os pressupostos para a utilização de testes paramétricos, recorreu-se ao teste de Kruskal-Wallis para verificar a existência de diferenças significativas entre os três grupos na avaliação inicial. Os resultados mostram que os grupos eram significativamente diferentes em todas as competências avaliadas: $(2)=39.92, p<.001$ para a Identificação de letras; (2) =37.01, p <.001 para a Escrita de letras; (2) =12.22, p = .002 para a Consciência fonémica; (2) = 15.38, $\mathrm{p}<.001$ para a Leitura de palavras e $(2)=11.82, p<.001$ para a Leitura de pseudopalavras.
Tabela 2 - Descrição dos resultados por tarefa entre os três grupos de crianças

\begin{tabular}{|c|c|c|c|c|c|c|c|}
\hline \multirow{2}{*}{ Tarefas } & \multirow{2}{*}{ Avaliação } & \multicolumn{2}{|c|}{ Grupo 1} & \multicolumn{2}{|c|}{ Grupo 2} & \multicolumn{2}{|c|}{ Grupo 3} \\
\hline & & $\mathbf{M}$ & DP & M & DP & $\mathbf{M}$ & DP \\
\hline \multirow{3}{*}{ Identificação de letras } & M1 & 17 & 10,2 & 62,5 & 15,2 & 57,3 & 18,5 \\
\hline & M2 & 87 & 16,8 & 82 & 11,6 & 68,6 & 20,3 \\
\hline & Ganho & 70 & & 19,5 & & 11,2 & \\
\hline \multirow{3}{*}{ Escrita de letras } & M1 & 6,3 & 5,1 & 54,6 & 16,4 & 57,5 & 32,3 \\
\hline & M2 & 85,5 & 18,3 & 92,4 & 14,5 & 74,9 & 30,4 \\
\hline & Ganho & 79,1 & & 37,8 & & 17,4 & \\
\hline \multirow{3}{*}{ Consciência fonémica } & M1 & 12,8 & 20,3 & 35,4 & 31 & 33,3 & 34,0 \\
\hline & M2 & 77,5 & 22,1 & 83,8 & 25,3 & 57,7 & 39,4 \\
\hline & Ganho & 64,7 & & 48,3 & & 24,4 & \\
\hline \multirow{3}{*}{ Leitura de palavras } & M1 & 0,5 & 2,3 & 18,9 & 29,3 & 18,8 & 23,3 \\
\hline & M2 & 83 & 11,7 & 62,2 & 32,9 & 37,6 & 41,0 \\
\hline & Ganho & 82,6 & & 43,3 & & 18,8 & \\
\hline \multirow{3}{*}{ Leitura de pseudopalavras } & M1 & $0^{*}$ & $0^{*}$ & 15,6 & 23,7 & 12,8 & 21,2 \\
\hline & M2 & 77,3 & 20,2 & 56,7 & 34,4 & 29,9 & 37,2 \\
\hline & Ganho & 77,3 & & 41,1 & & 17,1 & \\
\hline
\end{tabular}

* No Grupo 1, nenhuma criança conseguiu ler qualquer pseudopalavra

No sentido de perceber as diferenças entre os três grupos em comparação antes da intervenção, foram efetuados três testes de Mann-Whitney, com correção de Bonferroni ( $\mathrm{p}$ <.0167). Entre o Grupo 1 (intervenção mista) e o Grupo 3 (controlo), verificaram-se diferenças estatisticamente significativas na Identificação de letras, na Escrita de letras e na Leitura de palavras e na Leitura de pseudopalavras, sempre a favor do Grupo 3. Entre o Grupo 2 (intervenção Graphogame) e o Grupo 3 (controlo) não se verificou e existência de diferenças significativas em qualquer das provas. Comparando os dois grupos que seriam alvo de intervenção, isto é o Grupo 1 (intervenção mista) e o Grupo 2 (intervenção Graphogame), registam-se diferenças estatisticamente significativas em todas as provas (Tabela 3). 
Tabela 3 - Comparação dos desempenhos, por grupo, antes da intervenção (M1)

\begin{tabular}{|c|c|c|c|}
\hline & Grupo 1 vs Grupo 3 & Grupo 2 vs Grupo 3 & Grupo 1 vs Grupo 2 \\
\hline Identificação de letras & $\mathrm{U}=6,00, \mathrm{p}<.001$ & $\begin{array}{c}\mathrm{U}=109,50, \mathrm{p}=.229 \\
\mathrm{~ns}\end{array}$ & $\mathrm{U}=1,00, \mathrm{p}<.001$ \\
\hline Escrita de letras & $\mathrm{U}=21,00, \mathrm{p}<.001$ & $\begin{array}{c}\mathrm{U}=126,50, \mathrm{p}=.453 \\
\mathrm{~ns}\end{array}$ & $\mathrm{U}=.000, \mathrm{p}<.001$ \\
\hline Consciência fonémica & $\begin{array}{c}\mathrm{U}=99,00, \mathrm{p}=.029 \\
\mathrm{~ns}\end{array}$ & $\begin{array}{c}\mathrm{U}=117,00, \mathrm{p}=.320 \\
\mathrm{~ns}\end{array}$ & $\mathrm{U}=90,00, \mathrm{p}<.001$ \\
\hline Leitura de palavras & $\mathrm{U}=62,50, \mathrm{p}<.001$ & $\begin{array}{c}\mathrm{U}=117,00, \mathrm{p}=.311 \\
n s\end{array}$ & $\mathrm{U}=137,50, \mathrm{p}<.001$ \\
\hline Leitura de pseudopalavras & $\mathrm{U}=96,00, \mathrm{p}=.003$ & $\begin{array}{c}\mathrm{U}=125,00, \mathrm{p}=.424 \\
\mathrm{~ns}\end{array}$ & $\mathrm{U}=144,00, \mathrm{p}<.001$ \\
\hline
\end{tabular}

Após as dezasseis semanas de intervenção, os alunos foram sujeitos às mesmas provas. Recorreu-se igualmente ao teste de Kruskal-Wallis para verificar a existência de diferenças significativas entre os três grupos na segunda avaliação. Os resultados mostram que os grupos eram significativamente diferentes em todas as competências avaliadas: (2) $=11, .008, p=.004$ para a Identificação de letras; (2) $=6.284, p=.043$ para a Escrita de letras; $(2)=9.284, p=.011$ para a Consciência fonémica; $(2)=12,126, p=.002$ para a Leitura de palavras e $(2)=13.189, p=001$ para a Leitura de pseudopalavras. No sentido de perceber as diferenças entre os três grupos, foram efetuados três testes de Mann-Whitney, com correção de Bonferroni $(\mathrm{p}<.0167)$.

Comparando os grupos 1 (intervenção mista) e 3 (controlo), o sentido das diferenças que se registaram na Identificação de letras, na Leitura de palavras e na Leitura de pseudopalavras inverteu-se, passando a ser significativamente diferentes a favor do Grupo 1. Na Escrita de letras e na Consciência fonémica não se registaram diferenças estatisticamente significativas.
Entre o Grupo 2 (intervenção Graphogame) e o Grupo 3 (controlo) verificou-se, após a intervenção, a existência de diferenças significativas na Identificação de letras e na Escrita de letras a favor do Grupo 2. Em todas as outras competências o Grupo 2 apresentou resultados superiores ao Grupo 3, embora sem significância estatística.

Comparando agora os dois grupos alvo de intervenção - Grupo 1 (intervenção mista) e o Grupo 2 (intervenção Graphogame) - apenas se registam, na segunda avaliação, diferenças estatisticamente significativas na Consciência fonémica, na qual o Grupo 2 apresentou resultados superiores ao Grupo 1.

Tabela 4 - Comparação dos desempenhos, por grupo, após a intervenção (M2)

\begin{tabular}{lccc}
\hline & Grupo 1 vs Grupo 3 & Grupo 2 vs Grupo 3 & Grupo 1 vs Grupo 2 \\
Identificação de letras & $\mathrm{U}=68,00$, & $\mathrm{U}=72,00$, & $\mathrm{U}=151,00$, \\
& $\mathrm{p}=002$ & $\mathrm{p}=.015$ & $\mathrm{p}=.033 \mathrm{~ns}$ \\
Escrita de letras & $\mathrm{U}=114,50$, & $\mathrm{U}=60,50$, & $\mathrm{U}=193,00$, \\
& $\mathrm{p}=.092$ & $\mathrm{p}=.004$ & $\mathrm{p}=.250 \mathrm{~ns}$ \\
Consciência fonémica & $\mathrm{U}=120,00$, & $\mathrm{U}=78,50$, & $\mathrm{U}=120,00$, \\
& $\mathrm{p}=.115 \mathrm{~ns}$ & $\mathrm{p}=.024 \mathrm{~ns}$ & $\mathrm{p}=.002$ \\
Leitura de palavras & $\mathrm{U}=55,00$, & $\mathrm{U}=79,5$, & $\mathrm{U}=152,00$, \\
\multirow{2}{*}{ Leitura de pseudopalavras } & $\mathrm{p}<.001$ & $\mathrm{p}=.030 \mathrm{~ns}$ & $\mathrm{p}=.037 \mathrm{~ns}$ \\
& $\mathrm{U}=48,00$, & $\mathrm{U}=74,5$, & $\mathrm{U}=157,00$, \\
& $\mathrm{p}<.001$ & $\mathrm{p}=.019 \mathrm{~ns}$ & $\mathrm{p}=.050 \mathrm{~ns}$ \\
\hline
\end{tabular}

Os gráficos 1 e 2 permitem uma leitura clara do comportamento das diferentes competências nos dois momentos de avaliação (M1=pré-intervenção; M2=pós-intervenção) em todas as competências. 
Gráfico 1 - Resultados na Identificação de letras, na Escrita de letras e na Consciência fonémica por grupo e momento de avaliação

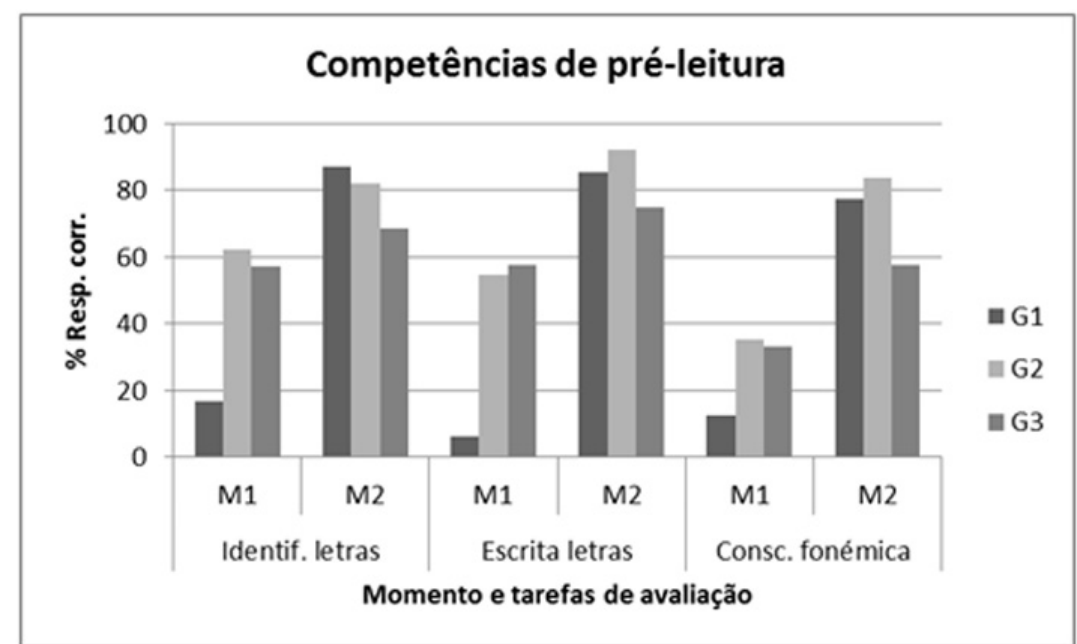

Gráfico 2 - Resultados na Leitura de palavras e de pseudopalavras por grupo e momento de avaliação

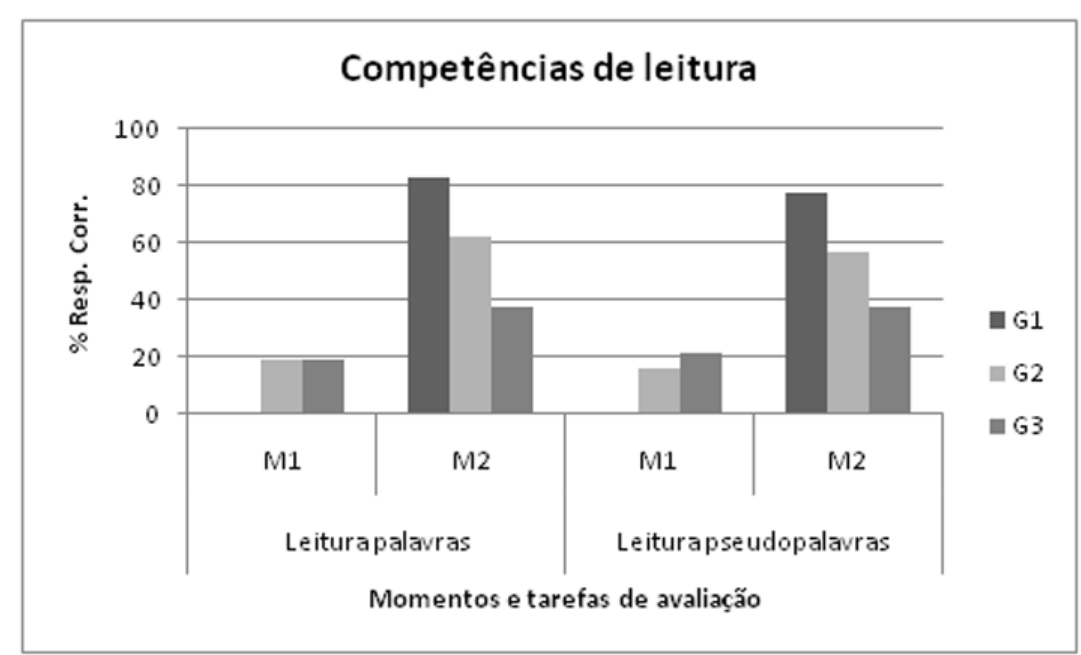

\section{Discussão}

O objetivo da leitura consiste no "processo de simultaneamente extrair e construir significado através da interação e envolvimento com a linguagem escrita" (RAND Reading Study Group, 2002, p. 11). Este objetivo não é diretamente atingível. De facto, é necessário o desenvolvimento de um conjunto de processos prévios e mais básicos como a descodificação. Por sua vez, para dominar o processo de descodificação a criança necessita de desenvolver a consciência fonémica e o conhecimento das relações letra-som.

A fase inicial da aprendizagem da leitura, também conhecida por fase alicerce, tem por base o conhecimento das relações entre letras e sons. No início dessa fase a adoção de correspondências entre letras e sons parece ocorrer em simultâneo com a emergência da consciência fonémica (DUNCAN, SEYMOUR e HILL, 1997; SEYMOUR E DUNCAN, 2001; SUCENA, 2005; SUCENA E CASTRO, 2011). O conhecimento das relações entre letras e sons, estreitamente relacionado com a consciência fonémica é necessário ao desenvolvimento dos dois processos cognitivos nesta fase: o processo logográfico e o processo alfabético.

O processo logográfico permite a identificação e armazenamento de um conjunto reduzido de palavras muito familiares, independentemente da sua complexidade ortográfica. Este processo consiste no reconhecimento de palavras baseado em sequências de letras mais ou menos complexas, desde uma primeira fase em que a atenção da criança se baseia em exclusivo na primeira letra, passando depois pela atenção à primeira e última letras, até finalmente serem incluídas no processo de análise as letras em posição medial (EHRI, 1992; STUART e COLTHEART, 1988; SEYMOUR e EVANS, 1999). O processo alfabético permite a descodificação sequencial de palavras e de pseudopalavras com ortografia simples, através do estabelecimento de um conjunto de relações simples entre letras e sons. 
A fase alicerce corresponde, entre as crianças inglesas, aos dois primeiros anos de escolaridade, e entre as crianças portuguesas ao início do terceiro trimestre do $1^{\circ}$ ano de escolaridade (SEYMOUR e EVANS, 1999; SUCENA, 2005; SERRANO et al., 2011). Como já referido, os dois processos - alfabético e logográfico - dependem do conhecimento de letras. Resultados obtidos com crianças falantes do português europeu revelam que, até ser atingido um conhecimento de cerca de $80 \%$ das letras, os resultados de leitura não atingem os $50 \%$ de respostas corretas em tarefas de leitura isolada de palavras. Após atingido esse limiar, observa-se uma nuvem de resultados de leitura acima dos 50\% (SUCENA, 2005; SERRANO et al. 2011).

A relação entre a literacia alicerce e a consciência fonológica expressase pela necessidade da consciência explícita da unidade fonema: tanto o processo logográfico como o alfabético se baseiam nesta unidade. Na sequência dos resultados de Ehri (1992), segundo os quais a aprendizagem do vocabulário visual se baseia nas correspondências grafema-fonema, outros autores (SEYMOUR e EVANS, 1999; Seymour et al. 2003; SHARE, 1995) propõem que o processo logográfico despoleta a consciência explícita da unidade fonémica. $O$ processo alfabético baseia-se na construção de relações entre letras e sons, por outras palavras, a criança adquire a noção de que cada letra representa um fonema, para o que a consciência fonémica explícita é também condição sine qua non (DUNCAN et al., 1997).

Como já referido, o sistema regular de ensino é eficiente com a generalidade das crianças no desenvolvimento dos processos básicos associados à aprendizagem da leitura. No entanto, no que respeita às crianças que evidenciam indicadores de futuras dificuldades de aprendizagem, o sistema nem sempre tem resultados satisfatórios. Efetivamente, as crianças que expressam dificuldade ao nível da consciência fonémica e das relações letra-som beneficiam de tempo intensivo de treino repetido que dificilmente pode ocorrer no contexto massificado de sala de aula.
O recurso ao Graphogame surge como uma resposta interessante na medida em que é potenciado o treino intensivo mas com caráter lúdico, sendo assim atenuada a frequente resistência da parte destas crianças em treinar competências que sentem ser exigentes. Os resultados do estudo piloto do Graphogame haviam já revelado um impacto positivo do treino diário em sessões de dez a quinze minutos realizadas em contexto escolar, sendo atingidos resultados significativamente mais elevados para o grupo experimental, quando comparados com o grupo de controlo. Mais especificamente, o grupo alvo de intervenção com o Graphogame obteve resultados francamente positivos ao nível das competências pré-leitoras. No entanto, ao nível das competências leitoras (leitura isolada de palavras e de pseudopalavras) apesar de a progressão ser significativa, os resultados não foram além dos 50\%. Importa enfatizar um aspeto relativo ao impacto do Graphogame que é a necessidade da presença de um técnico de educação que supervisione o decorrer do jogo de modo a solucionar pequenas dificuldades técnicas e, mais importante, a aperceber-se de dificuldades em processos específicos parte da criança e que não estão a ser ultrapassados com base unicamente no software. No estudo piloto não foi efetuada uma intervenção mista, já que, por questões metodológicas importava avaliar o impacto do recurso, em exclusivo, à ferramenta Graphogame.

No presente estudo foi explorado o impacto de uma intervenção que combina o recurso ao Graphogame com o treino (igualmente com carater lúdico) em sessões presenciais das mesmas competências trabalhadas pelo Graphogame (pré-leitoras e leitoras). Foi desenvolvida uma intervenção com um grupo de crianças com recurso diário exclusivo ao software Graphogame e com um segundo grupo de crianças uma intervenção com recurso ao mesmo software (quatro vezes por semana) mas a que acrescia uma sessão semanal de promoção de competências pré-leitoras e leitoras orientada por um técnico de educação. 
Com base nos resultados recentes da investigação e da implementação do Graphogame, partiu-se para o presente estudo hipotetizando que uma intervenção combinada, com recurso ao Graphogame e a sessões estruturadas e sistemáticas de promoção das competências pré-leitora e leitoras teriam um efeito mais substancial sobre a progressão das crianças do que a intervenção com recurso exclusivo ao Graphogame. Foram avaliadas competências préleitoras (consciência fonémica e relações letra-som) e competências leitoras (leitura de palavras e leitura de pseudopalavras). Os resultados revelaram, para todas as competências, à exceção da Consciência fonémica, ausência de diferenças entre os dois grupos de intervenção. Estes resultados refletem a eficácia de qualquer das intervenções, confirmando o impacto positivo de jogar o Graphogame com periodicidade diária em contexto escolar. Em paralelo, os resultados refletem o impacto altamente positivo da combinação do Graphogame com uma sessão presencial de promoção da leitura. Importa salientar que o apoio presencial decorreu apenas uma vez por semana, em grupos de duas a cinco crianças, em contexto escolar. Isto é, os resultados obtidos a partir do recurso ao Graphogame podem ser potenciados com um aumento muito reduzido dos recursos humanos.

A não semelhança dos grupos no momento pré intervenção e o facto de não estarem reunidos os pressupostos para a utilização de testes não paramétricos não permite a efetuação de uma análise de covariância a fim de proceder a uma análise da eficiência do programa controlando as competências iniciais. Assim sendo, os resultados dos testes Mann-Whitney efetuados traduzem, de forma muito lacunar, os efeitos da intervenção. Ao nível das competências pré-leitoras (identificação e escrita de letras e consciência fonémica) os resultados dos grupos de intervenção 1 e 2 são muito próximos (em torno dos $80 \%$ ) e precisamente no limiar a partir do qual se torna possível a consolidação do processo de descodificação. Igualmente de relevar é a distância entre os grupos 1 e 2 no que respeita às competências leitoras (ca. 20\%), que se traduz em resultados entre os $50 \%$ e os $60 \%$ para o grupo de intervenção exclusiva com o Graphogame enquanto o grupo de intervenção mista obteve resultados de ca. $80 \%$ em ambas as tarefas.

Portugal tem índices de insucesso na aprendizagem da leitura e da escrita que urgem ser minorados, nomeadamente através da identificação precoce de crianças em risco, e da adoção combinada do Graphogame com sessões presenciais em pequeno grupo. Antecipar a intervenção até ao 10 ano, antes ainda de as dificuldades da criança se terem declarado por completo, aumenta a probabilidade de sucesso já que previne sentimentos de frustração altamente desmotivadores e geradores de afastamento em relação às tarefas escolares.

\section{Conclusões}

Na língua inglesa estão já identificadas diversas propostas de intervenção eficazes no apoio às dificuldades de aprendizagem da leitura, propostas essas assentes nos resultados da investigação. Na língua portuguesa o panorama é radicalmente diferente, pois escasseiam as propostas estruturadas e baseadas no conhecimento científico de apoio às dificuldades de aprendizagem da leitura disponíveis às comunidades educativa e clínica.

Como já referido, os programas de apoio à aprendizagem da leitura com melhores resultados combinam o treino explícito ao nível da consciência fonológica com instrução da leitura altamente estruturada (e.g., HATCHER, HULME e ELLIS, 1994; HATCHER et al., 2006). Especificamente, importa, numa primeira etapa, promover o treino explícito da consciência fonémica e das relações letra-som de modo a tornar possível o desenvolvimento do processo de descodificação. Os resultados deste estudo revelam que o software Graphogame Português Alicerce é uma ferramenta altamente eficaz a este nível. Idealmente, em paralelo com o recurso ao Graphogame, deve ser 
realizada uma intervenção pedagógica de modo a assegurar que a criança domina efetivamente as relações letra-som e que treina intensivamente o processo de descodificação. 0 facto de os três grupos não serem, à partida, equivalentes, aliado à impossibilidade de proceder a análises de covariância com controlo das competências iniciais por não se encontrarem satisfeitos os pressupostos para a utilização de testes paramétricos, constitui uma das limitações deste estudo, que se espera poder ultrapassar num estudo posterior e com uma amostra mais alargada.

Com o presente estudo espera-se não apenas preencher uma lacuna ao nível da intervenção precoce nas dificuldades de aprendizagem, mas também incentivar ao desenvolvimento de mais programas de intervenção cientificamente sustentados.

\section{Referências}

BLOK, Henk; OOSTDAM, Ron; OTTER, Martha; OVERMAAT, Marianne. Computer-assisted instruction in support of beginning reading instruction: A review. Review of Education Research, v. 72, p. 101-130, 2002.

DUNCAN, Lynne; SEYMOUR, Philip; HILL, Shirley. How important are rhyme and analogy in beginning reading? Cognition, v. 63, p. 171-208, 1997.

ELBRO, Carsten; RASMUSSEN, Ingelise; SPELLING, Birgitte. Teaching reading to disabled readers with language disorders: A controlled evaluation of synthetic speech feedback. Scandinavian Journal of Psychology, v 37, p. 140-155, 1996.

EHRI, Linnea. Reconceptualizing the development of sight word reading. In: GOUGH, Philip; EHRI, Linnea; TREIMAN, Rebecca (Eds.). Reading acquisition. Hillsdale, NJ: Erlbaum, 1992. p. 107-143

FOORMAN, Barbara; FRANCIS, David; FLETCHER, Jack; SCHATSCHNEIDER, Christopher; MEHTA, Paras. The Role of Instruction in Learning to Read: Preventing Reading Failure in At-Risk Children. Journal of Educational Psychology, v. 90, n. 1, p. 37-55, 1998.

HATCHER, Peter; HULME, Charles; ELLIS, Andrew. Ameliorating early reading failure by integrating the teaching of reading and phonological skills: The phonological linkage hypothesis. Child Development, v. 65, p. 41-57, 1994.
HATCHER, Peter; HULME, Charles; SNOWLING, Margaret. Explicit phoneme training combined with phonic reading instruction helps young children at risk of reading failure. Journal of Child Psychology and Psychiatry, University of York, UK, v. 45, n 2, p. 338-358, 2004

HATCHER, Peter; HULME, Charles; MILES, Jeremy; CARROLL, Julia; HATCHER, Janet; GIBBS, Simon; SMITH, Glynnis; BOWYER-CRANE, Claudine; SNOWLING, Margaret. Efficacy of small group reading intervention for beginning readers with reading-delay: a randomised controlled trial. Journal of Child Psychology and Psychiatry, v. 47, n. 8, p. 820-827, Aug. 2006.

HUEMER, Sini; LANDERL, Karin; ARO, Mikko; LYYTINEN, Heikki. Training reading fluency among poor readers of German: many ways to the goal. Annals of Dyslexia, v. 58, n. 2 , p. $115-137$, Dec. 2008.

JIMÉNEZ, Juan, HERNÁNDEZ-VALLE, Isabel; RAMÍREZ, Gustavo; ROSSARIO, Maria; RODRIGO, Mercedes; ESTÉVEZ, Adelina, O'SHANAHAN, Isabel; GARCÍA, Eduardo; TRABAUE, María. Computer speech-based remediation for Reading disabilities: The size of spelling-to-sound unit in a transparent orthography. The Spanish Journal of Psychology, v. 10, p. $52-67,2007$.

KYLE, Fiona; KUJALA, Janne; RICHARDSON, Ulla; LYYTINEN, Heikki; GOSWAMI, Usha. Assessing the Effectiveness of Two Theoretically Motivated Computer Assisted Reading Interventions in the United Kingdom: GG Rime and GG Phoneme. Reading Research Quarterly, v. 48, n. 1, p. 61-76, Jan. 2013.

LYNCH, Lisa; FAWCETT, Angela; NICOLSON, Roderick. Computer-assisted reading intervention in a secondary school: an evaluation study. British Educational Communications and Technology Agency, v. 31, p. 333-348, 2000.

LYYTINEN, Heikki. State-of-Science Review: SR-D12 New Technologies and Interventions for Learning Difficulties: Dyslexia in Finnish as a Case Study. Foresight Mental Capital and Wellbeing Project: The Government Office for Science. London: UK. 2008.

LYYTINEN, Heikki; RONIMUS, Miia; ALANKO, Anne; POIKKEUS, Anna-Maija; TAANILA, Maria. Early identification of dyslexia and the use of computer game-based practice to support reading acquisition. Nordic Psychology, v. 50, n. 6, p. 109-126, June 2007.

LYYTINEN, Heikki; ERSKINE, Jane; KUJALA, Janne; OJANEN, Emma; RICHARDSON, Ulla. In search of a science-based application: A learning tool for reading acquisition. Scandinavian Journal of Psychology, v. 59, n. 2, p. 668-675, Dec. 2009. 
LOVETT, Maureen; BARRON, Robin; BENSON, Nancy. Effective remediation of word identification and decoding difficulties in school-age children with reading disabilities. In: SWANSON, Lee; HARRIS, Karin; GRAHAM, Steve (Eds.). Handbook of learning disabilities. New York: Guilford, 2003. p. 273-292.

MAGNAN, Annie; ECALLE, Jean. Audio-visual training in children with reading disabilities. Computers \& Education, v. 46, p. 407-425, 2006.

RAND Reading Study Group. Reading for understanding: Toward an R\&D program in reading comprehension. Santa Monica, CA: RAND Corporation, 2002.

REGTVOORT, Anne; VAN DER LEIJ, Aryan. Early intervention with children of dyslexic parents: Effects of computer-based reading instruction at home on literacy acquisition. Learning and Individual Differences, v. 17, p. 35-53, 2007.

SAINE, Nina; LERKKANE, Marja-Kristiina; AHONEN, Timo; TOLVANEN, Asko; LYYTINEN, Heikkii. Computer-Assisted Remedial Reading Intervention for School Beginners at Risk for Reading Disability. Child Development, v. 82, n. 3, p. 1013-1028, Mar. 2011.

SERRANO, Francisca; GENARD, Nathalie; SUCENA, Ana; DEFIOR, Sylvia; ALEGRIA, Jesus; MOUSTY, Philippe; LEYBAERT, Jacqueline; CASTRO, São Luís; SEYMOUR, Philip. Variations in Reading and Spelling Acquisition in Portuguese, French and Spanish: A cross-linguistic comparison. Journal of Portuguese Linguistics, v. 10, n. 1, p. 183-204, 2011.

SEYMOUR, Philip; DUNCAN, Lynne. Learning to read in English. Psychology: The Journal of the Hellenic Psychological Society, v. 8, p. 281-299, 2001.

SEYMOUR, Philip; EVANS, Henryka. Foundation level dyslexias: Assessment and treatment. Journal of Learning Disabilities, v. 32, p. 394-405, 1999.

SEYMOUR, Philip; ARO, Mikko; ERSKINE, Jane. Foundation literacy acquisition in European orthographies. British Journal of Psychology, v. 94, p. 143-174, 2003.

SHARE, David. Phonological recoding and self-teaching: sine qua non of reading acquisition. Cognition, v. 55, n. 2, p. 151-218, 1995.

SIMÕES, Mário Rodrigues. Investigações no âmbito da aferição nacional do Teste das Matrizes Progressivas Coloridas de Raven (M.P.C.R.). Lisboa: Fundação Calouste Gulbenkian, 2003.

SOE, Kyaw; KOKI, Stan; CHANG, Juvenna. Effect of computer-assisted instruction (CAI) on reading achievement: A meta-analysis. Pacific Resources for Education and Learning, 2000. STUART, Morag; COLTHEART, Max. Does reading develop in a sequence of stages? Cognition, v. 30, p. 139-181, 1988.
SUCENA, Ana. Aprendizagem da leitura e da escrita em português europeu numa perspectiva trans-linguística. 2005. 456fls. Dissertação (Doutorado em Psicologia) Faculdade de Psicologia e de Ciências da Educação, Porto, 2005.

SUCENA, Ana; CASTRO, São Luís; SEYMOUR, Philip. Developmental dyslexia in an orthography of intermediate depth: the case of European Portuguese. Reading and Writing, v. 22, p. 791-810. 2009.

SUCENA, Ana; CASTRO, São Luís. ALEPE - Avaliação da Leitura em Português Europeu. Lisboa: CEGOC-TEA, 2011

SUCENA, Ana; SILVA, Ana; CRUZ, Joana; Viana, Fernanda. Portuguese Foundation Graphogame: Preliminary Results. Proceedings of the 17th European Conference on Developmental Psychology. No prelo.

TORGESEN, Joseph. Recent discoveries from research on remedial interventions for children with dyslexia. In: SNOWLING, Margaret; HULME, Charles (Eds.). The science of reading: A handbook. Oxford, UK: Blackwell, 2005. p. 521-537.

VAN DAAL, Victor; REITSMA, Pieter. Computer-Assisted learning to read and spell: Result from two pilot studies. Journal of Research in Reading, v. 23, p-181-193, 2000.

VELOSO, João. A língua na escrita e a escrita da língua. Algumas considerações gerais sobre transparência e opacidade fonémicas na escrita do português e outras questões. Da investigação às práticas: estudos de natureza educacional, v. VI, n 49, p. 49-68. 2005

VIANA, Fernanda Leopoldina; SUCENA, Ana. A avaliação da leitura. Necessidades, complexidades e instrumentos. In: OLIVEIRA, Jaima Pinheiro; BRAGA, Tânia Moron Saes; VIANA, Fernanda Leopoldina (Orgs.). Alfabetização em países de língua portuguesa: pesquisa e intervenção. São Paulo: Editora CRV. p. 67-84.

WIMMER, Heinz; MAYRINGER, Heinz. Dysfluent reading in the absence of spelling difficulties: A specific disability in regular orthographies. Journal of Educational Psychology, v. 94, p. 272-277, 2002.

WISE, Barbara; RING, Jerry; OLSON, Richard. Training phonological awareness with and without explicit attention to articulation. Journal of Experimental Child Psychology, v. 72, p. 271-304, 1999

Recebido em 25/04/2016.

Aceito em 03/07/2016. 